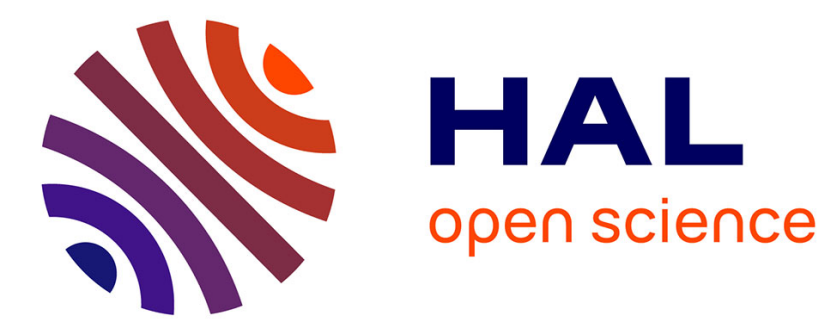

\title{
Les contrats économiques de souveraineté, outils de la régulation de la concurrence (les pratiques des autorités de concurrence à partir de l'exemple de la France) Laurence Boy
}

\section{- To cite this version: \\ Laurence Boy. Les contrats économiques de souveraineté, outils de la régulation de la concurrence (les pratiques des autorités de concurrence à partir de l'exemple de la France). Revue Internationale de Droit Economique, 2010, 3/2010, pp.271-297. hal-00721570}

\section{HAL Id: hal-00721570 \\ https://hal.science/hal-00721570}

Submitted on 6 Aug 2012

HAL is a multi-disciplinary open access archive for the deposit and dissemination of scientific research documents, whether they are published or not. The documents may come from teaching and research institutions in France or abroad, or from public or private research centers.
L'archive ouverte pluridisciplinaire HAL, est destinée au dépôt et à la diffusion de documents scientifiques de niveau recherche, publiés ou non, émanant des établissements d'enseignement et de recherche français ou étrangers, des laboratoires publics ou privés. 


\title{
Les contrats économiques de souveraineté, outils de la régulation de la Concurrence (les pratiques des autorités de concurrence à partir de l'exemple de la France)
}

\author{
Laurence Boy ${ }^{1}$
}

\begin{abstract}
Résumé: Dans le cadre concurrentiel devenu mondial, concentration et organisation de l'économie résultent on ne peut plus clairement de deux facteurs : le jeu combiné des stratégies des acteurs privés et publics mais aussi les moyens proposés par le système juridique pour les accompagner et/ou les encadrer.

Les procédures négociées illustrent la notion proposée par certains de contrat économique, en l'espèce, de contrat de souveraineté, outils de régulation de la concurrence. Ce texte propose une étude de droit économique de ces contrats de structuration et de restructuration du capital dans la régulation du droit de la concurrence. Les analyses conduisent à s'intéresser, au-delà des strictes obligations juridiques traditionnelles et détaillées souscrites par les entreprises, à l'équilibre général de l'accord, au but économique qu'il poursuit. Il s'agit, en effet, d'une convention globale formant un tout cohérent en dépit des dispositions diverses qu'elle peut contenir, ce qui explique en grande partie son régime juridique. En échange d'engagements pour rendre l'opération ou les comportements compatibles avec une concurrence praticable, autorités publiques et juges négocient dans ces contrats leur souveraineté. Même si la menace de sanction pèse toujours, contre une certaine indulgence, les entreprises s'engagent à respecter l'ordre public concurrentiel. On assiste ainsi substantiellement à une organisation conjointe et concertée de la concentration et du pouvoir économique, sous la houlette des fameuses Autorités administratives indépendantes, sous contrôle du juge. La souplesse caractéristique des engagements des entreprises s'accompagne corrélativement d'une vigilance dans le suivi des contrats économiques en droit de la concurrence. Le facteur temps est essentiel en droit économique. Dans les procédures négociées, les autorités de concurrence ont donc pris l'habitude de faire appel à des mandataires (trustees) pour suivre non seulement les engagements comportementaux mais aussi structurels qui, plus simples à exécuter a priori, soulèvent eux aussi de sérieuses difficultés, comme en témoignent des affaires récentes. L'efficacité et l'efficience des engagements tiennent sans doute à ce qui est à l'origine du contrat : la confiance et la réciprocité.
\end{abstract}

1 Introduction

2 Les «engagements» en droit de la concurrence, un exemple type de contrats économiques

2.1 Les «engagements» du droit de la concurrence s'inscrivent dans des contrats économiques

2.2 Les «engagements» en droit de la concurrence, des contrats de souveraineté (la décision administrative et/ou la justice négociée)

3 Nature originale des contrats économiques de souveraineté en droit de la concurrence

3.1 Des obligations variées ordonnées autour d'une unicité de l'objet du contrat: la restructuration contrôlée du capital

3.2 Une nature complexe ordonnée autour du facteur temps

Summary

1 Professeur à l'Université de Nice-Sophia Antipolis, CREDECO/GREDEG UMR 6227 CNRS/INRA. 


\section{Introduction}

Le droit de la concurrence a toujours été au cœur du droit économique. Si le droit de la concurrence visait et/ou vise encore dans de nombreux pays le contrôle des prix et des aides d'Etats, il a pour objet principal de sanctionner le respect d'un ordre public concurrentiel et, désormais, d'encadrer l'organisation de l'économie menée par les pouvoirs privés, qu'elle résulte des opérations de concentrations, de pratiques concertées ou unilatérales.

Présenté à l'origine principalement comme un doit répressif, il fait largement appel de nos jours à la régulation, au point qu'il paraît légitime de parler de régulation de la concurrence plutôt que de droit de la concurrence ${ }^{2}$. La régulation ${ }^{3}$ est une préoccupation fondamentale des pouvoirs publics et plus spécifiquement des diverses autorités de concurrence. Même si ce n'est pas la concurrence pure et parfaite mais une concurrence raisonnable (reasonable) ou praticable (workable), voire en « trompe-l'œil » qui est visée, ce n'est qu'assez récemment que les autorités de concurrence ont été dotées aux Etats-Unis ${ }^{5}$, en Europe ainsi que dans les différents Etats européens et en voie de développement $^{6}$ des outils propres de cette régulation. Elle repose sur divers mécanismes caractéristiques d'un droit souple allant de l'autodiscipline ${ }^{7}$ à l'égard d'objectifs concurrentiels (compliance) à la régulation confiée à un tiers: une agence (Autorité Administrative Indépendante, AAI) ou un juge.

Avec la «régulation de la concurrence», il s'agit d'associer la souplesse nécessaire dans les réactions des autorités aux pratiques néfastes des entreprises aux politiques publiques et, parfois mais pas nécessairement, l'ouverture de certains secteurs à la concurrence. La régulation de la concurrence est ainsi plus large que la seule notion de régulation sectorielle ${ }^{8}$. Il est généralement admis aujourd'hui que la régulation c'est aussi la régulation de la concurrence ${ }^{9}$.

Depuis son apparition, cette idée de régulation de la concurrence n'a fait que se renforcer. La pratique des autorités de concurrence en fait l'instrument juridique par excellence de leurs politiques. Elles affirment désormais ouvertement rechercher à la fois tant la lutte contre les pratiques les plus graves que l'efficacité de la «répression». L'utilisation de la répression était souvent aveugle, reconnaissent-elles. La démonstration de certaines infractions, qui doit être aussi respectueuse des droits de l'homme et du droit procédural, ne permet pas toujours de «frapper» là où les pratiques sont les plus graves et ne tient pas nécessairement compte des responsabilités réelles des acteurs. La répression n'est pas nécessairement pédagogique. L'exemplarité de la peine

\footnotetext{
* Professeur à l’Université de Nice Sophia Antipolis, CREDECO/GREDEG UMR 6227 CNRS/INRA.

2 L. Boy, « Le droit de la concurrence : régulation et/ou contrôle des restrictions de concurrence », Conférence à l'Institut européen de Florence, décembre 2003, JCP 2004, I, 166 et Contrats, Concurrence et Consommation nov. 2004, $\mathrm{n}^{\circ} 11$, p. 16; B. Lasserre, Propos Introductifs au colloque sur Clémence et transaction en matière de concurrence: premières expériences et interrogations de la pratique, colloque CREDA, Paris, 19 janvier 2005 , disponible sur : http://www.creda.ccip.fr/colloques/pdf/2005-clemence-transaction/actes-clemence.pdf; sur l'évolution, H. Ullrich, « Competitor Cooperation and the Evolution of Competition Law : Issues for Research in a Perspective of Globalization », in J. Drexl (ed.) The Future of Transnational Antitrust - from Comparative to Common Competition Law, Kluwer Law International, 2003, p. 159.

${ }^{3}$ G. J. Martin (dir.), les transformations de la régulation juridique, LGDJ, coll. Droit et société, 1998 ; L. Boy; «Réflexions sur 'le droit de la régulation'», Dalloz 2001, Chr., p. 3031.

${ }^{4}$ F. Riem, «Concurrence effective ou concurrence efficace? L'ordre concurrentiel en trompe-l'œil », RIDE 2008, $\mathrm{n}^{\circ} 1$, p. 67.

5 E. Fox, R. Peritz \& L. Sullivan, U.S. Antitrust in Global Context: Cases and Materials, West/Thomson, 2004 and Supp. 2009; D. Geradin \& M. Rato, "Frand Commitments and EC Competition Law", 2009, disponible sur: http://papers.ssrn.com/sol3/papers.cfm?abstract_id=1527407.

${ }^{6} \mathrm{P}$. Debrosse, «Les programmes de clémence à l'épreuve de la globalisation des marchés », RIDE 2010, nº 2, p. 211.

${ }^{7}$ On doute du qualificatif de régulation qui suppose selon nous, l'intervention d'un tiers.

${ }^{8}$ M.-A. Frison-Roche, Le droit de la régulation, Dalloz 2001, p. 610. Contra: L. Boy, « Réflexions sur 'le droit de la régulation' », op. cit..

${ }^{9}$ Lors du colloque Clémence et transaction en matière de concurrence : premières expériences et interrogations de la pratique, précité, le président Lasserre affirmait haut et fort que le Conseil de la concurrence fait de la régulation; L. Boy, « Réflexions sur 'le droit de la régulation'», op. cit.
} 
doit faire place à la connaissance réelle et à la correction effective des comportements, utilisant à cette fin une morale nouvelle de type utilitariste.

A la répression a été substituée l'idée de régulation de la concurrence qui implique non seulement des actions réactives de type punitif mais aussi et surtout des actions préventives et d'accompagnement de type pédagogique. L'efficacité de la politique de concurrence passe par deux voies complémentaires : un renforcement des pouvoirs de sanction des autorités de concurrence pour les pratiques les plus graves et, paradoxalement, le développement des programmes de clémence, de transaction et d'engagements ${ }^{10}$ qui peuvent constituer, en outre, un avantage probatoire.

Comme le relevait Bruno Lasserre, président du Conseil de la concurrence, aujourd'hui président de l'Autorité de concurrence, ces dernières années ont vu la montée en puissance des A.A.I. (agencies) et des procédures de clémence (leniency) ${ }^{11}$. Les moyens de réguler la concurrence offerts au Conseil et aujourd'hui à l'Autorité de concurrence interne ${ }^{12}$ comme aux autorités communautaires ou étrangères sont nombreux. Il en est ainsi principalement du contrat au sens large avec notamment la pratique des engagements apparue d'abord en droit de la concentration puis introduite progressivement dans le droit des pratiques anticoncurrentielles. Le contrat visé ici n'est cependant pas le simple contrat du Code civil. C'est un contrat économique, mieux, un contrat économique de souveraineté ${ }^{13}$.

La notion de contrat économique n'a pas été assez développée dans la doctrine ${ }^{14}$. Elle est essentielle cependant pour expliquer de nombreux phénomènes «contractuels » contemporains mais que la doctrine classique a du mal à appréhender. Cette notion bouscule, en effet, trop d'habitudes de pensée du modèle juridique libéral, spécialement dans la doctrine française car c'est là que la formalisation des conséquences de ce droit a été poussée à l'extrême, notamment par l'institution d'un dualisme juridictionnel. Paradoxalement, la connaissance du droit français libéral dans son formalisme le plus achevé apparaît comme la meilleure école en faveur de l'analyse substantielle ${ }^{15}$, par la mise en lumière des traits caractéristiques du droit libéral (séparation public/privé, contrat/acte unilatéral, objet des obligations, formation/effets du contrat...).

Les contrats économiques existent depuis longtemps déjà. Leur existence semble avoir précédé leur essence, aurait dit le doyen Vedel ${ }^{16}$. Certains de ces contrats étaient déjà utilisés depuis des années, même si ce n'était pas sous ce vocable ${ }^{17}$. Ils ont fait l'objet de véritables analyses sous l'angle du

\footnotetext{
${ }^{10}$ Ch. Gheur \& N. Petit, Alternative Enforcement Techniques in EC Competition Law: Settlements, Commitments and Other Novel Instruments, Bruxelles, Bruylant, 2009; D. Kling, « Clémence et transaction en matière de concurrence », Gazette du Palais, $\mathrm{n}^{\circ} 287$ et 288, 14 et 15 octobre 2005, p. 5.

${ }^{11}$ Propos introductifs au colloque précité, p. 7; S. Holmes et Ph. Girardet, The International Comparative Legal Guide to Cartels and Leniency, Global Legal Group Ltd, 3rd ed. revised, 2010, disponible sur: http://www.iclg.co.uk/khadmin/Publications/pdf/3380.pdf.

${ }_{12}$ Ordonnance du 13 novembre 2008, JORF $\mathrm{n}^{\circ} 0265 \mathrm{du} 14$ novembre 2008.

${ }^{13}$ Le concept théorique est développé dans : «Les procédures négociées en droit de la concurrence : les retours d'expériences analysés par les juristes et les économistes», in 4èmes rencontres de droit économique du CREDECO, juin 2010, à paraître dans Les Dossiers de la RIDE.

${ }^{14}$ Contra: G.J. Martin, Cours inédit de droit économique, Nice, 2004/2005; T. L. Anderson, D. P. Parker, "Sovereignty, Credible Commitments, and Economic Prosperity on American Indian Reservations", The Journal of Law and Economics, vol. 51, nov. 2008. L'expression "contrats économiques" est utilisée dans le droit chinois: "Economic Contract Law" (Adopted at the fourth session of the Fifth National People's Congress on December 13, 1981, amended in accordance with the "Decision on Revision of the 'Economic Contract Law of the People' s Republic of China'", adopted by the third session of the Eighth National People's Congress Standing Committee on September 2, 1993): art 2: "This law is applicable to contracts signed between legal persons who are equal civil parties (dont sont exclues les personnes publiques), other economic organizations, self-employed workers or traders and rural households operating on contract for the purpose of realizing certain economic goals and clarifying each other's rights and obligations».

${ }^{15}$ G. Farjat, «L'importance d'une analyse substantielle en droit économique», RIDE 1986, n0, p. 9; J.B. Racine et F. Siiriainen, « Retour sur l'analyse substantielle », RIDE 2007, n 3, p. 259; CREDECO, Le droit économique aujourd'hui, Dalloz, 2010, p. 1436.

${ }^{16}$ G. Vedel, «Le droit économique existe-t-il ? », in Mélanges Vigreux, coll. Travaux et recherches de l'IPA-IAE de Toulouse, tome II, 1981, p. 767 et suiv.

${ }^{17}$ On peut en trouver les premières traces dans les divers modes de gestion déléguée des services publics.
} 
droit économique après les «décolonisations» ${ }^{18}$, notamment sous le nom de contrats $\mathrm{d}^{\prime}$ 'investissement ${ }^{19}$. Les pays nouvellement décolonisés avaient un cruel besoin d'investissements pour assurer le développement du pays et recouraient, pour ce faire, à des contrats d'investissement passés avec des entreprises étrangères souvent aussi puissantes, voire plus puissantes qu'eux ${ }^{20}$. Ils n'avaient généralement pas le choix, et l'objet de ces contrats était bien un objet économique : organiser le développement économique de leur pays, d'une branche ou d'un secteur d'activité. L'examen de ces contrats retrouve aujourd'hui un intérêt évident tant en droit international qu'interne avec le développement extraordinaire des PPP (partenariats publics-privés, privatepublic partnerships). Ils ont toujours présenté un grand intérêt en droit interne : contrats de cessions d'entreprises (qu'elles soient in bonis ou en difficultés - fusions, acquisitions, droit des procédures collectives $^{21}-$ ), contrats d'organisation de réseaux d'intégration variés ${ }^{22}$, contrats d'engagements divers dans le droit de la concurrence. C'est à ces derniers que l'on souhaite s'attacher en raison de leur double intérêt. D'une part, ils sont une illustration particulièrement exemplaire du concept même de contrat économique, d'autre part, ils constituent une catégorie originale qui met en lumière le caractère très relatif des oppositions traditionnelles du droit libéral, notamment entre la sphère publique de l'intérêt général et la sphère privée des intérêts privés.

\section{Les «engagements» en droit de la concurrence, un exemple type de contrats économiques}

Plusieurs tentatives de classification des contrats économiques ont été proposées. Malgré leur diversité, on retiendra qu'elles ont en commun la définition même du contrat économique et l'existence d'une catégorie bien identifiée, celle des contrats de souveraineté.

Pour définir les contrats économiques, G. J. Martin enseigne ${ }^{23}$ que ce sont ceux qui mettent en oeuvre les «rapports économiques», c'est-à-dire les rapports qui se superposent aux rapports patrimoniaux classiques et qui ont pour objet la concentration et/ou l'organisation de l'activité économique. Sur le plan méthodologique, la référence ainsi faite à l'objet doit retenir toute l'attention. C'est en effet un terme essentiel dans la théorie du contrat. L'hypothèse de travail retenue et à laquelle nous nous rallions est que sont des contrats économiques ceux dont l'objet (au sens du droit des contrats) est la concentration et/ou l'organisation de l'économie. Encore faut-il savoir ce que l'on désigne par l'objet du contrat. Or cet objet est double ou du moins doit être dédoublé. Il faut, en effet, différencier soigneusement l'objet du contrat de l'objet des obligations des parties ${ }^{24}$.

\footnotetext{
${ }^{18}$ En Asie et surtout en Afrique dans les années 1960.

${ }^{19}$ R. Charvin, L'investissement international et le droit au développement, préface S. Regourd, L'harmattan 2002 ; G. Blanc, Le contrat international d'équipement industriel, Thèse Aix 1980; A. Mezghani, « La signification du prix dans les contrats clé en main », J.D.I. n 2, 1990, p. 271 ; J. Touscoz, Assurances et garanties des opérations internationales de commerce et d'investissement, évolutions ou révolution ?, D.P.C.I. 1990, Tome 16, nº 1, p. 112.

20 D.E. Vielleville1, B.S. Vasani, "Sovereignty over Natural Resources versus Rights under Investment Contracts: which one prevails?", Transnational Dispute Management (TDM), Vol. 5, Issue 2 , April 2008, disponible sur: http://www.crowell.com/documents/Sovereignty-Over-Natural-Resources-Versus-Rights-Under-Investment-

Contracts Transnational-Dispute-Management.pdf : «By the end of the 20th century, the pendulum had swung once again. The 1980s and 1990s were characterized by the growing interest of developing nations in receiving foreign investment by means of new projects or privatizations of already existing state-owned enterprises. Added to this, many nations agreed to enter into bilateral investment treaties and multilateral agreements to promote themselves as investment-friendly countries».

${ }^{21} \mathrm{~N}^{\circ}$ spécial « Entreprises en difficultés et concurrence », RIDE, 1995, $\mathrm{n}^{\circ} 2$.

${ }^{22}$ G.J. Martin, «Les contrats d'intégration dans l'agriculture », RTD com. 1974, p. 271; L. Boy, « L'inadaptation de la loi du 6 juillet 1964 à la protection réelle des agriculteurs intégrés », note sous Grenoble, 27 janvier 1981, Dalloz 1982, p. 625 .

${ }^{23}$ Cours précité.

${ }^{24}$ Selon le droit anglo-saxon, "consideration is the legal concept of value in connection with contracts. It is anything of value in the common sense, promised to another when making a contract. It can take the form of money, physical objects, services, promised actions, abstinence from a future action and much more”. Voir: http://en.wikipedia.org/wiki/Contract. Il s'agit d'un concept voisin de l'objet ou de la cause des obligations, deux
} 
L'objet du contrat lui-même et qui autorise sa qualification de contrat économique est son objet global qui est toujours la concentration ou l'organisation de l'économie. La loi chinoise précise ainsi que l'objet de ces contrats est «de réaliser certains objectifs économique» ${ }^{25}$. Nous verrons qu'il se distingue de l'objet des obligations des parties, lesquelles sont extrêmement précises et qui relèvent de l'analyse plus traditionnelle de l'objet telle qu'elle est faite généralement par la doctrine (objet des obligations).

L'organisation de l'économie a été souvent l'objet propre de la planification de l'économie par l'Etat, que ce soit de façon autoritaire (les économies dites planifiées des économies socialistes) ou de façon incitative ${ }^{26}$. Elle est toujours le fait, de façon plus inavouée et subtile, des pouvoirs privés économiques eux-mêmes ${ }^{27}$ via les nombreux contrats et montages conventionnels juridiques (groupes de sociétés, réseaux contractuels de distribution, d'intégration amont, sous-traitance, etc.). "La grande firme moderne et l'appareil moderne de la planification socialiste sont deux variantes qui expriment la même adaptation à un même besoin.» ${ }^{28} \mathrm{La}$ planification est aussi souvent organisée de façon concertée par les pouvoirs privés et publics. C'est ici que l'on peut parler de recours aux contrats économiques de souveraineté dans la régulation de la concurrence ${ }^{29}$.

En effet, s'il l'on s'interroge sur de nombreuses pratiques mises en oeuvre par les autorités de concurrence dans le cadre du contrôle des concentrations et du contrôle des pratiques anticoncurrentielles (antitrust) et faisant appel à la technique générale des engagements, on peut légitimement les qualifier de contrats économiques et, plus spécifiquement encore, de contrats de souveraineté. Le propos peut choquer a priori. Il doit donc être expliqué.

\subsection{Les «engagements» du droit de la concurrence s'inscrivent dans des contrats économiques}

L'objet traditionnel du droit de la concurrence au sens général est le contrôle de la concentration ${ }^{30}$ ainsi que des pratiques anticoncurrentielles: ententes et abus de domination ayant des effets anticoncurrentiels ${ }^{31}$. C'est dire que les autorités de la concurrence doivent veiller à l'application de ce droit autoritaire qui prévoit soit un contrôle préventif (un système administratif d'autorisation préalable pour les opérations de concentration) soit un contrôle a posteriori mettant en jeu des sanctions avant tout administratives et répressives ou même pénales.

Les procédures d'engagements modifient sensiblement ce schéma. A la «décision» verticale et venue d'en haut, qu'elle soit administrative, quasi juridictionnelle ou juridictionnelle, se substitue un mécanisme plus complexe dans lequel prennent place la discussion, la concertation, l'échange

\footnotetext{
concepts voisins en droit français au point que Planiol envisageait de supprimer le concept de cause qu'il trouvait inutile car faisant doublon avec l'objet (M. Planiol, Traité élémentaire de droit civil, t. II, Paris, Librairie Cotillon, $1900, \mathrm{n}^{\circ}$ 77/78). En revanche, il ne vise pas non plus ce que l'on désigne par objet du contrat et qui permet d'envisager l'opération dans son ensemble et de la qualifier juridiquement. Marty et Raynaud, Les obligations, Tome 2, vol. 1, Sirey $1962, n^{\circ} 164$ sur la cause catégorique qui rejoint l'objet du contrat; Overstake, Essai de classification des contrats spéciaux, Thèse Bordeaux, L.G.D.J. 1969.

${ }^{25}$ Loi précitée du 13 décembre 1981.

${ }^{26}$ On pense à «l'ardente» obligation évoquée par le Général de Gaulle, qualificatif qui n'est pas à proprement juridique.

${ }^{27}$ J.K. Galbraith, Le nouvel Etat industriel, Gallimard, NRF,1967. L'auteur consacre un chapitre entier à la planification privée des entreprises destinée à s'affranchir des incertitudes du marché intitulé: «La nature de la planification industrielle».

${ }^{28}$ J.K. Galbraith, op. cit., p. 45.

${ }^{29}$ A. Perrot, «Le modèle du contrat dans les nouvelles conceptions des régulations économiques », in M.-A. FrisonRoche (ed.), Droit et économie de la régulation, t.4, Les engagements dans les systèmes de régulation, Paris, Presses de Sciences Po/ Dalloz, 2006, p. 147. L'auteur parle de «contrats de régulation».

${ }^{30}$ Clayton Act paragraphes 7 \& 7A; Règlement UE n ${ }^{\circ}$ 4064/89 du Conseil, 21 déc. 1989, JOCE n L 395, 30 déc. 1989 , tel que modifié par le règlement $\mathrm{n}^{\circ}$ 1310/97 du Conseil, 30 juin 1997, JOCE n L 180, 9 juill. 1997.

${ }^{31}$ Sherman Act; Cases and Materials on European Community Law de R. J. Goebel, W. J. Davey, E. M. Fox et G. A. Bermann, West Publishing Co, 1999; Private Enforcement of Antitrust Law in the EU, UK and USA de Clifford Jones et John Temple Lang, Oxford University Press 1999 ; Articles 101 et 102 TFUE; articles L 420-1 et L 420-2 du Code français du commerce.
} 
des consentements, plus ou moins libres, d'ailleurs ${ }^{32}$, entre les entreprises et les autorités de concurrence. L'objet de ces discussions résulte de la volonté des pouvoirs publics, pour des raisons d'efficacité déjà évoquées, non d'interdire purement et simplement des pratiques ou des opérations de restructuration mais de les accompagner et de les encadrer dans un sens voulu par les pouvoirs publics, qui est globalement le respect d'une concurrence praticable, laquelle constitue la boussole de l'ordre public libéral. L'idée est de concilier liberté de la concurrence et liberté contractuelle, valeurs qui entrent souvent en conflit, comme en témoigne le droit des ententes. Ces deux libertés autorisent les entreprises à rechercher une organisation conventionnelle de l'économie ainsi qu'un certain pouvoir économique, voire social via leur pouvoir de marché. Ces libertés ne doivent cependant pas violer l'ordre public économique ${ }^{33}$ libéral et ce qui constitue aujourd'hui l'ordre public néo-libéral : l'ordre public concurrentiel ${ }^{34}$.

L'ordre public a toujours été l'un des points cardinaux du droit des contrats ${ }^{35}$. A ce titre, le recours aux «engagements» dans le droit de la concurrence devait nécessairement interroger le juriste sur le contenu de cet ordre public concurrentiel. On sait que l'ordre public économique contemporain est de plus en plus positif (et non plus seulement prohibitif) et qu'il entend orienter les contrats. Il s'agit d'un ordre public souvent de direction, à côté d'un ordre public de protection ${ }^{36}$ dont les exemples types étaient constitués par le droit du travail ${ }^{37}$ et le droit de la consommation ${ }^{38}$. En réalité, l'unité fondamentale de l'ordre public économique résulte selon nous de la fonction instrumentaliste de ce dernier: l'Etat entend plus ou moins autoritairement orienter les comportements en raison des nécessités de sa politique économique. Cette observation nous paraît fondamentale au regard des engagements en matière de droit de la concurrence : elle souligne l'une des difficultés de la matière qui est de conduire une véritable «politique» économique tout en garantissant aux opérateurs privés un minimum de prévisibilité et de sécurité juridique ${ }^{39}$, valeurs essentielles, voire prioritaires de tout système juridique ${ }^{40}$.

La souplesse associée à la sécurité juridique que donne le contrat (pacta sunt servanda est le credo mondial du droit contractuel) sont vite apparues comme le vecteur principal des politiques générales de recherche de l'efficacité des autorités de concurrence. Via ses agences et autorités

\footnotetext{
${ }^{32}$ Les praticiens insistent généralement sur la pression qu'exercent les autorités de la concurrence sur les entreprises et qui altèrerait fondamentalement la liberté de ces dernières plus ou moins contraintes de «passer par les fourches caudines» des gardiens du droit de la concurrence. C. Lemaire, «Engagements - Art. 6CEDH: La Cour d'Appel de Paris se prononce sur l'incidence de la nature spécifique de la procédure d'engagements sur les droits des parties (Canal 9/GIE Les indépendants) », Concurrences 2009, $\mathrm{n}^{\circ} 1$, p. 181; C. Lucas de Leyssac, « À propos des situations non fautives de non-competition », Concurrences 2006, $\mathrm{n}^{\circ} 4$, p. 1.

${ }^{33}$ G. Farjat, L'ordre public économique, préface B. Goldman, LGDJ, 1963.

${ }^{34}$ Le concept a été proposé par A. Pirovano. Voir à ce sujet: M. Rainelli, «L'ordre concurrentiel: approche d'un économiste », L. Boy ; «L'ordre concurrentiel, essai de définition d'un concept »; F. Riem, « Droits de la concurrence et ordre concurrentiel »; H. Ullrich, «L'ordre concurrentiel, rapport de synthèse », in Droit économique et sécurité juridique, éd. Frison-Roche, 2003, pp. 13, 33, 635 et 663, respectivement.

${ }^{35} \mathrm{Ph}$. Malaurie, L'ordre public et le contrat, Thèse Paris, 1951; JL. Bergel, Méthodologie juridique, PUF, Thémis, 2001, p. 184 : 'Notion cadre', intentionnellement vague et qui résiste à toute définition précise, il concerne les domaines homogènes tels que l'ordre public de l'Etat, l'ordre public processuel, l'ordre public pénal, l'ordre public économique et social...et l'ordre public civil»; Demogue estimait qu'il était le «bâti» de la société (Les notions fondamentales de droit privé. Pour servir d'introduction à l'étude des obligations, La mémoire du droit, 2002) et M. Portalis écrivait : «Le maintien de l'ordre public dans une société est le but suprême. Protéger des conventions contre cette loi, ce serait placer des volontés particulières au-dessus de la volonté générale. Ce serait dissoudre l'Etat», «Exposé des motifs du titre préliminaire du Code Napoléon », in Ecrits et discours juridiques et politiques, PUAM, Aix-en-Provence, 1988, p. 78.

${ }^{36}$ Cette distinction proposée par le doyen Carbonnier et reprise par G. Farjat (précité) est loin d'être absolue selon nous.

${ }^{37}$ Sur la remise en cause du droit du travail par le culte du marché: A. Supiot, L'esprit de Philadelphie, La justice sociale face au marché, Seuil, coll. Idées, 2010.

${ }^{38}$ J. Calais-Auloy et F. Steinmetz, Droit de la consommation, Dalloz 2006.

${ }^{39}$ L. Boy, J-B. Racine, F. Siiriainen (dir.), Sécurité juridique et droit économique, Bruxelles, Larcier, 2008,586 p.

${ }^{40}$ N. Molfessis, Combattre l'insécurité juridique ou la lutte du système juridique contre lui-même, Rapport public du Conseil d'Etat 2006, p. 391, disponible sur : http://lesrapports.ladocumentationfrancaise.fr/BRP/064000245/0000.pdf ; L. Boy, J.-B. Racine, F. Siiriainen (dir.), Sécurité juridique et droit économique, op. cit.
} 
administratives indépendantes, l'Etat cherche moins à prohiber les comportements des acteurs économiques qu'à obtenir leur adhésion à une politique concurrentielle évolutive, qu'il s'agisse de l'ouverture à la concurrence (la théorie de facilités essentielles mobilise dès le départ le «contrat plus ou moins forcé») ou de la gestion des «crises» (elles ont justifié un peu partout le concept d'entreprise défaillante dans le contrôle des opérations de concentration). Bref, il s'agit d'organiser de concert avec les entreprises privées l'économie nationale ou régionale, ce qui ne va d'ailleurs pas toujours sans difficultés (la taille optimale des champions doit-elle être nationale ou européenne? ${ }^{41}$. On sent immédiatement comment la doctrine classique peut être choquée par ces procédures contractuelles. Dans un monde généralement éthéré de «parties supposées égales» et visant principalement à l'échange de biens et de droits considérés comme «l'équivalent voulu», se superposent des contrats dont l'objet est de contrôler, sous l'égide des autorités de la concurrence, l'organisation concurrentielle de nos économies. Ces conventions sont d'autant plus étonnantes qu'elles associent pouvoirs publics et pouvoirs privés, et surtout qu'elles autorisent les pouvoirs publics à négocier l'une des formes de leur souveraineté : la décision administrative «sanctionatrice», voire la décision de justice et qui plus est, parfois la justice répressive, l'un des symboles de la souveraineté étatique. De telles conventions permettraient à l'Etat de négocier sa souveraineté, en tout cas de remettre en cause la séparation du politique et de la société civile.

\subsection{Les «engagements» en droit de la concurrence, des contrats de souveraineté (la décision administrative et/ou la justice négociée)}

En France, les interrogations sur la nature réellement contractuelle des instruments de régulation de la concurrence concernent tant les privatistes que les publicistes, qu'il s'agisse des engagements devenus «classiques» ${ }^{42}$ en droit de la concentration, de la clémence, de la non-contestation des griefs ou des diverses formes de transaction, dont la vraie transaction européenne en droit de la concurrence $^{43}$. Sont rejetées tant la nature contractuelle de ces derniers que celle de contrat pouvant porter sur la souveraineté de l'Etat.

Pour les premiers, le dogme de l'autonomie de la volonté comme celui de l'égalité contractuelle («qui dit contractuel dit juste? On pourrait gloser à l'envi sur ce terrain...» ${ }^{44}$ ) s'opposent à l'idée même que ces «engagements» puissent recevoir la qualification de contrat. Commentant l'affaire qui a fait le plus de bruit ces derniers temps en France, celle dite du «cartel de l'acier», un auteur se demandait si la procédure française de non-contestation des griefs est bien une «procédure négociée» ${ }^{45}$. "Le système mis en place par l'article L 464-2 III du Code de commerce ne laisse guère de place à la négociation avec le collège: les parties sont simplement 'entendues', le résultat de la négociation est simplement 'proposé' par le Rapporteur, celui-ci ne lie pas l'Autorité. Et l'on pourra encore ajouter que depuis la réforme introduite par l'ordonnance de 2008, la noncontestation des griefs est déconnectée de la prise d'engagements. Pour la non-contestation proprement dite, il n'y a donc plus rien à négocier (...)» (souligné par nous). S'agissant de la transaction européenne, l'auteur insiste sur le «fait qu'en réalité, cette procédure n'est pas une procédure négociée (...) La procédure est déséquilibrée (souligné par nous), ce qui jure avec le concept contractuel, par hypothèse égalitaire». Il s'agirait, malgré les termes, d'un acte purement unilatéral $^{46}$.

\footnotetext{
${ }^{41}$ L. Idot, Entre concurrence et retour de l'Etat : les Etats « schyzos » ?, Concurrences, $2008 \mathrm{n}^{\circ} 4$, éditorial.

42 A. Pappalardo, «Les nouvelles règles en matière de contrôle communautaire des concentrations d'entreprises », RIDE 1998, n², p. 177.

43 OCDE, Experience with Direct Settlements in Cartels Cases, Oct. 2009, p. 69, disponible sur: http://www.oecd.org/dataoecd/11/9/44178372.pdf.

${ }^{44}$ D. Bosco, «Transaction européenne, non-contestation des griefs française, filles perdues? », in $4^{e}$ rencontres de droit économique du CREDECO, précité.

${ }^{45}$ Ibid.

${ }^{46}$ Ibid. Contra: L. Boy, « Cartel de l'acier: des sanctions pour une méthodologie exemplaire », Rev. Lamy Concurrence, avril/juin 2010, p. 21 et s, spéc. p. 23.
} 
Rejeter l'idée selon laquelle les procédures diverses d'engagements relèveraient d'une nature contractuelle au motif qu'elles sont inégalitaires nous paraît totalement irréaliste. A s'en tenir à une telle conception égalitaire du contrat, il faudrait incontestablement dénier une telle nature à tous les contrats de consommation dont les innombrables contrats d'adhésion qui jalonnent la vie de tout un chacun. Or, malgré les analyses de Saleilles ${ }^{47}$ qui proposait d'y voir en partie des règlements unilatéraux d'origine privée pour en contrôler la «légalité», la doctrine a toujours considéré qu'ils devaient être analysés comme de simples contrats ${ }^{48}$. La volonté doit être distinguée en droit du consentement. Il s'agit d'une volonté juridique qui est une épure de la volonté psychologique ${ }^{49}$ et, comme le dit avec beaucoup de réalisme M.-A. Frison-Roche, consentir, c'est se soumettre en partie $^{50}$. La nature conventionnelle de ces pratiques ne nous semble guère pourvoir être contestée. On trouve pourtant le même refus de qualification contractuelle non plus des privatistes mais d'une bonne partie de la doctrine de droit public et ce, pour une raison totalement incompatible avec la précédente et fondée sur un dogme: celui de la toute-puissance de l'Etat et de l'interdiction qui lui serait faite de négocier sa souveraineté. Là où il y a souveraineté, il ne saurait qu'y avoir pouvoir de police ou acte juridictionnel public ${ }^{51}$. Lorsque les autorités de la concurrence utilisent les voies des engagements conventionnels, elles resteraient malgré tout dans l'exercice de prérogatives publiques insusceptibles de recours à l'outil contractuel.

De telles analyses sont totalement dépassées elles aussi et ne correspondent pas à la réalité ${ }^{52}$, déjà ancienne d'ailleurs en droit de l'arbitrage, qui est celle de la reconnaissance des contrats de souveraineté, c'est-à-dire de contrats dans lesquels l'Etat accepte, plus ou moins librement lui aussi $^{53}$, de renoncer à sa force souveraine formelle et de négocier sur un pied de relative égalité avec des partenaires privés.

De nombreux contrats de souveraineté sont conclus régulièrement quoiqu'en dise une certaine doctrine administrative française. On peut le regretter éventuellement pour diverses raisons. Cela ne doit pas interdire de les étudier. L'on constate depuis longtemps un abandon de souveraineté du pouvoir juridictionnel dans le droit international avec la validité de l'arbitrage d'Etat. Ici encore, les solutions françaises sont exemplaires car c'est le pays où l'hostilité à la soumission des personnes publiques à l'arbitrage a sans doute été la plus marquée... de la part du juge administratif mais pas de celle de la Cour de cassation. Malgré la coutume internationale qui, au nom de la souveraineté étatique, refuserait la compétence de tout autre juge (a fortiori l'arbitre) que celui de l'Etat en cause avec une partie privée, très tôt des conventions d'arbitrage ont été conclues directement entre Etats et particuliers ${ }^{54}$, et l'arbitrage international s'est rapidement imposé dans le commerce international comme le mode normal de règlements des différends, y compris dans les pays du Tiers-Monde soucieux de protéger au départ leur souveraineté nouvellement conquise ${ }^{55}$. En France, cependant, l'opposition à l'arbitrabilité des litiges où les pouvoirs publics sont en cause est demeurée vive,

\footnotetext{
${ }^{47}$ R. Saleilles, La théorie générale de l'obligation, La Mémoire du Droit, 2001; G. Farjat, Droit privé de l'économie, PUF, Thémis, 1975; L. Boy, «Les 'utilités' du contrat », Les Petites Affiches, 10 septembre 1997, n 109, p. 3.

${ }^{48}$ J. Ghestin (dir.), G. Goubeaux, avec le concours de M. Fabre-Magnan, Traité de droit civil, Introduction générale, $4{ }^{\mathrm{e}}$ éd., 1994; V. Forray, Le consensualisme dans la théorie générale du contrat, LGDJ, 2007, «L'Acte juridique », recueil d'articles, in Droits, n $^{\circ}$ 7, P.U.F., Revue française de théorie juridique, 1988.

${ }^{49} \mathrm{G}$. Rouhette, Contribution à l'étude critique de la notion de contrat, Thèse Paris, 1965.

${ }^{50}$ M.-A. Frison-Roche, «Distinction de la volonté et du consentement », RTD Civ. 1995, p. 373; Ph. Coppens, « Sur l'intention et la volonté dans les contrats », in Mélanges M. Fontaine,Bruxelles, Larcier, 2003, p. 83.

${ }^{51}$ De Laubadère, F. Moderne, Delvolvé, Traité des contrats administratifs, Tome II, LGDJ, 1986, ${ }^{\circ} 1728$.

${ }^{52}$ Y. Madiot, Aux frontières du contrat et de l'acte administratif unilatéral : recherches sur la notion d'acte mixte en droit public français, LGDJ, Bibl. dr. public, 1972, t. 103.

${ }^{53}$ Dans certains cas, les entreprises privées sont plus puissantes que les Etats avec lesquels elles contractent.

${ }^{54}$ Entreprises et Etats (France, UK) et Egypte pour le canal de Suez au XIXéme siècle; G. Guyomar, «L'arbitrage concernant les rapports entre Etats et particuliers », in Annuaire français de droit international, volume 5, 1959, pp. 333-354.

${ }^{55}$ La Cour de cassation française a été l'une des premières à affirmer l'arbitrabilité des litiges étatiques et la séparabilité de la clause compromissoire. A. Mezghani, Droit international privé, Tunis, Cérès Productions, 1991; Abi-Saab, "The Newly Independent States and the Rules of International Law: An Outline", 8 HOW. L.J. 1962, p. 99; R.-P. Anand, New States and International Law, Vikas publishing House Pvt. Ltd, 1972. Depuis ont été institués des centres d'arbitrage institutionnels comme le CIRDI dont l'objectivité n'est pas contestée.
} 
comme en témoigne la position du Conseil d'Etat dans la convention complexe d'investissement avec la société Walt Disney Productions en 1986. Par dérogation à l'interdiction de principe posée par l'article 2060 du Code civil à l'encontre des collectivités publiques de compromettre ${ }^{56}$, le législateur a dû adopter un texte législatif «de circonstance» pour autoriser le recours à l'arbitrage.

La «neutralisation du pouvoir d'Etat» ${ }^{57}$ ne s'observe pas seulement en ce qui concerne le pouvoir juridictionnel de l'Etat; elle a été parfaitement décrite dans le domaine des contrats économiques avec les clauses dites de stabilisation ou d'intangibilité par lesquelles l'Etat, soucieux d'attirer les investissements étrangers, garantit à l'opérateur privé de stabiliser, voire d'écarter les dispositions de droit fiscal ou de droit social ${ }^{58}$, par exemple. On ne signalera sans doute jamais assez l'importance des contrats de souveraineté dans l'analyse des contrats économiques et, plus généralement, des contrats économiques de souveraineté (contrats d'investissement, partenariats public/privé ${ }^{59}$ ). Dans ces derniers, afin de concourir à ou d'encadrer l'activité économique conjointement avec les pouvoirs privés économiques, l'Etat accepte généralement de neutraliser ses pouvoirs normatifs et juridictionnels.

S'agissant de restructuration du capital, le phénomène de neutralisation du pouvoir d'Etat est peutêtre plus récent sans doute pour deux raisons. Le droit de la faillite a d'abord longtemps été considéré comme une affaire purement privée entre un débiteur et ses créanciers, dans laquelle l'Etat ne jouait que son rôle traditionnel de juge tranchant en tant qu'autorité extérieure à un conflit d'intérêts privés ${ }^{60}$. Avec l'importance prise par certaines faillites et leurs conséquences tant économiques (disparition de branches d'activité ou de secteurs) que sociales, la volonté de sauvetage des entreprises s'est accompagnée d'un rôle croissant et plus dynamique des pouvoirs publics, notamment du juge, dans le droit des procédures collectives via les plans de continuation et de sauvetage divers ${ }^{61}$. On ne laisse plus aux seules entreprises faillies et repreneurs potentiels le soin de restructurer le capital défaillant (anciens concordats, cessions à forfait): le juge, en contrôlant lui-même la cessation d'activité ou la reprise d'activité(s), s'immisce par un acte dont la nature est discutée en doctrine (un jugement mais empruntant en partie à l'accord de volonté) dans l'organisation de l'économie. Le juge devient selon l'heureuse formule un «juge-entraîneur» ${ }^{62}$. Conscient de ce rôle dans la restructuration du capital défaillant, notamment dans les «crises» successives du libre marché, le législateur a imposé d'ailleurs un minimum de dose de concurrence dans un domaine où régnait une grande opacité. Les restructurations du capital visibles ou

\footnotetext{
${ }^{56}$ Il a fallu le vote d'un texte conjoncturel pour autoriser le recours à l'arbitrage en l'espèce: Art. 9 de la loi du 19 août 1986 portant diverses dispositions relatives aux collectivités locales, M. de Boissesson, «Interrogations et doutes sur une évolution législative: l'article 9 de la loi du 19 août 1986 », Rev. de l'arbitrage, 1987, p. 3; L. Boy, «L'arbitrabilité des litiges internationaux concernant les personnes morales de droit public en droit français (contribution de droit économique) », RIDE, 1992, n² 2, p. 99.

${ }^{57} \mathrm{P}$. Mayer, « La neutralisation du pouvoir normatif de l'Etat en matière de contrats d'Etat », JDI, 1986, p. 5.

${ }^{58}$ Ibid.; P. Weill, «Principes généraux du droit et contrats d'Etats », in Droit des relations économiques internationales : études offertes à Berthold Goldman, Paris 1982, p. 387; J.F. Lalive, Contrats entre Etats ou entreprises publiques et personnes privées, Cours de La Haye, Tome 181, p. 296. L'Etat français a ainsi renoncé dans son contrat avec Walt Disney Productions à certaines dispositions du droit français du travail et du droit de l'urbanisme; T. C. Daintith, «Contract Design and Practice in the Natural Resources Sector », in The Complex Long-term Contract : Structures and International Arbitration, Fritz Nicklisch, Heidelberg, 1987, pp. 151-169.

${ }^{59}$ Voir notamment: Règlement (CE) n ${ }^{\circ} 2195 / 2002$ du Parlement européen et du Conseil du 5 novembre 2002 relatif au vocabulaire commun pour les marchés publics (CPV), JO, $\mathrm{n}^{\circ} \mathrm{L} 340$ du 16 déc 2002, p. 0001-0562; Directive 2004/18/CE du Parlement européen et du Conseil, du 31 mars 2004, relative à la coordination des procédures de passation des marchés publics de travaux, de fournitures et de services, JO, L 134 du 30 avril 2004, p. 114-240; Directive 2004/17/CE du Parlement européen et du Conseil, du 31 mars 2004, portant coordination des procédures de passation des marchés dans les secteurs de l'eau, de l'énergie, des transports et des services postaux, JO, L 134 du 30 avril 2004, p. 1-113.

${ }^{60}$ Sur l'office traditionnel du juge: S. Guinchard et alii, Droit processuel, Droit commun et droit comparé du procès équitable, Dalloz, $6^{\mathrm{e}}$ éd. 2009; L. Cadiet, J. Normand, S. Amrani Mekki, Théorie générale du procès, PUF, Thémis, $2010, \mathrm{n}^{\circ} 220$ et $\mathrm{s}$.

${ }^{61}$ A. Pirovano (dir.), Changement social et droit négocié, Economica, 1988.

${ }^{62}$ F. Ost, «Jupiter, Hercule, Hermès: trois modèles du juge », in P. Bouretz, La force du droit, éd. Esprit, coll. Philosophie, 1991, p. 241.
} 
invisibles ${ }^{63}$ s'opèrent tous les jours dans le droit de la concurrence. Qu'il s'agisse de fusions, de prises de capital, de constitutions de réseaux ou d'ententes, elles intéressent les pouvoirs publics qui ont longtemps considéré ces dernières exclusivement sous l'angle des prohibitions. Dans tous les pays dotés d'un droit de la concurrence ont été ou sont introduites des procédures d'engagements par lesquelles l'autorité de concurrence/le juge recourt, dans le but d'orienter les pratiques vers la politique économique voulue, à des engagements avec les entreprises négociant ainsi sa souveraineté administrative et/ou juridictionnelle pour des raisons d'efficacité et de gain procédural $^{64}$.

Mieux vaut prendre acte de cette réalité et l'observer plutôt que de la nier car des problèmes restent à résoudre, qu'il s'agisse du régime global des «contrats» ou de la qualification juridique dont dépendent certaines solutions concrètes comme le suivi de décisions négociées.

\section{Nature originale des contrats économiques de souveraineté en droit de la concurrence}

La principale originalité des contrats économiques tient, comme G.J. Martin l'a souligné, à la superposition aux obligations «traditionnelles» des parties, d'un objet général : l'organisation de l'économie et la concentration du capital. Ils nécessitent donc une analyse «à double détente» afin de mettre en lumière cette volonté organisatrice. Cette première analyse est rendue plus complexe encore avec les engagements qui nous intéressent du fait que ce sont aussi des contrats de souveraineté. En effet, l'organisation de l'économie se fait entre pouvoirs privés et pouvoirs publics qui s'engagent pour ces derniers à négocier leur souveraineté. Avec les engagements du droit de la concurrence, l'objet économique du contrat consiste à encadrer la volonté de gagner ou de conserver un pouvoir de marché excessif et/ou à contrôler les restructurations du capital qui peuvent avoir des conséquences néfastes sur la concurrence en général et, in fine, dit-on, le bien-être des consommateurs. Contrats économiques passés entre les entreprises et les autorités de concurrence, leur nature soulève de grandes difficultés que peut sans doute aider à résoudre la démonstration de la complexité de l'opération formalisée dans les engagements.

\subsection{Des obligations variées ordonnées autour d'une unicité de l'objet du contrat: la restructuration contrôlée du capital}

Si la doctrine ne s'intéresse généralement pas à l'étude de l'objet des contrats économiques, il ne faut pas pour autant avoir une attitude inverse et négliger l'analyse des obligations des parties. Au premier abord, elles consistent pour ces dernières à s'engager à donner, à faire ou à ne pas faire quelque chose (ce qui relève de l'échange classique ou de la production). Cet objet traditionnel des obligations des parties ${ }^{65}$ n'a pas de sens en lui-même pour nous renseigner sur l'objet économique du contrat. Ce n'est, en effet, pas autour de l'objet éclaté des diverses obligations des parties que s'organise l'économie générale du contrat. Mais c'est en mettant en perspective ces diverses obligations et leurs objets que se révèle l'objet final du contrat qui, lui, est toujours un objet "économique", rendant compte au-delà des opérations d'échange ou de production, de l'objectif fondamental poursuivi. Il est donc indispensable d'étudier ces obligations et leur articulation qu'il s'agisse de celles des entreprises ou

\footnotetext{
${ }^{63}$ Certaines intégrations très poussées sont à cet égard exemplaires: l'intégré reste formellement propriétaire de ses moyens de production - c'est lui qui court le risque de l'entreprise et le risque du crédit - mais a perdu la propriété substantielle de ces derniers, notamment la liberté de la gestion de ces biens, de décider des modes d'exploitation et de gestion de ces derniers.

${ }^{64}$ Les textes sont déjà anciens qui portent en droit américain comme en droit européen sur la négociation de la sanction même en ce qui concerne le noyau dur du droit pénal (Plea guilty, et plus récemment en France comparution sur reconnaissance préalable de culpabilité).

65 Voir, sur la notion de consideration, note 24 ; également, http://en.wikipedia.org/wiki/Consideration_in_English_law.
} 
de celles des autorités de concurrence : ne pas poursuivre, voire ne même pas qualifier l'opération d'infraction aux règles de concurrence ${ }^{66}$.

Même si la technique des engagements est relativement récente en droit de la concurrence ${ }^{67}$, la doctrine a dégagé quelques grands types d'engagements pris par les firmes : les engagements structurels, comportementaux et quasistructurels.

Les nombreuses procédures de «clémence» au sens large du terme infirment de façon définitive le seul rôle répressif du droit de la concurrence. Il semble, en outre, que le cadre traditionnel du droit de la concurrence fondé principalement sur la distinction structures (concentration)/comportements soit en partie remise en question ${ }^{68}$. Les moyens de réguler la concurrence offerts aux autorités de concurrence internes comme communautaires sont extrêmement nombreux: clémence stricto sensu, transaction, engagements, mise en conformité (compliance $)^{69}$. Certes, les autorités disposaient déjà de la possibilité de prononcer des astreintes et surtout des injonctions variées aux entreprises ${ }^{70}$. Ces dernières surtout permettent généralement aux autorités d'avoir une véritable politique de régulation de la concurrence. En effet, ce sont non seulement des injonctions d'abstention (mettre fin à des pratiques $)^{71}$ mais aussi des injonctions de modification des comportements des entreprises ${ }^{72}$. Les véritables innovations ont conduit à la possibilité pour les autorités américaines puis européennes et nationales d'encourager les entreprises parties à une infraction supposée à collaborer elles-mêmes à leur établissement ou à leur cessation avant même toute qualification juridique d'infraction. Il s'agit, entre autres, tant la terminologie est variable, de la clémence et/ou de la non-contestation des griefs $^{73}$. L'idée qui préside aux «engagements» au sens large est que «la régulation nécessite surtout des outils flexibles, des outils plus souples, tels que des recommandations, des avis, le règlement des litiges, l'élaboration de catalogues de bonnes pratiques; elle associe davantage les acteurs du marché et privilégie la discussion, la médiation, les compromis négociés» ${ }^{74}$.

\footnotetext{
${ }^{66}$ Procédure de non-contestation des griefs en droit français, nolo contendere en droit américain. Il ne s'agit pas techniquement d'une reconnaissance de culpabilité, mais elle est souvent offerte comme une partie des négociations entre le procureur et l'avocat de la défense, incluant parfois le juge, pour réduire la gravité des charges.

${ }^{67}$ J. E. Kwoka, L. J. White, The Antitrust Revolution: Economics, Competition, and Policy, Oxford University Press, 2004; M.-C. Boutard-Labarde, G. Canivet et alii, L'application en France du droit des pratiques anticoncurrentielles, LGDJ, 2008.

${ }^{68}$ L. Idot, « De quelques illustrations des limites de la distinction entre le comportemental et le structurel », Revue des contrats, $1^{\text {er }}$ juillet $2009, \mathrm{n}^{\circ} 3$, p. 1070.

${ }^{69}$ D. Kling, «Clémence et transaction en matière de concurrence », Gazette du Palais, n 287 et 288,14 et 15 oct. 2005, p. 5, W. J. Kolasky1, Deputy, Assistant Attorney General Antitrust Division U.S. Department of Justice, Before the Corporate Compliance, 2002, Conference Practising Law Institute (PLI), San Francisco, CA, July 12, 2002.

${ }^{70}$ Pour la France, par exemple: article L 464-2, I, al 1er du Code de commerce.

${ }^{71}$ Mettre fin à une entente (déc. n 9-D-49, 24 juin 1997, BOCCRF, 17 sept. 1997); modifier le comportement d'une entreprise en position dominante (déc. $\mathrm{n}^{\circ} 87 \mathrm{D}-08,18$ avril 1987, BOCCRF, 15 mai 1987).

${ }^{72}$ Déc. $\mathrm{n}^{\circ}$ 02-D-44, 11 juil. 2002, BOCCRF, 30 sept. 2002. Ces injonctions aboutissent à remodeler les conventions intervenues entre les entreprises; R. Poésy, Aspects procéduraux du droit français des pratiques anticoncurrentielles, Thèse Nice, 2000.

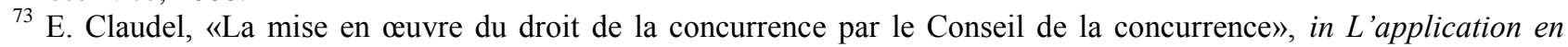
France du droit des pratiques anticoncurrentielles, LGDJ, 2008, p. 481; E. Chevrier, «Synthèse sur les engagements en matière de concurrence», D., 2008, p. 1044; A. Vialfont, «Le droit de la concurrence et les procédures négociées», RIDE, 2007, n², p. 157; A. de Streel, «Introduction », Reflets et perspectives de la vie économique, 1/2008 (Tome XLVII), p. 5; L. Idot, «Le régime des engagements en cours de formalisation », Revue des contrats, $1^{\mathrm{er}}$ juillet 2005 $\mathrm{n}^{\circ}$ 3, p. 697; B. Lasserre, «La non-contestation des griefs en droit français de la concurrence: bilan et perspectives d'un outil pionnier », Concurrences, 2008, $\mathrm{n}^{\circ} 2, \mathrm{n}^{\circ} 17870$, pp. 93-100; F. Zivy, « La procédure de non-contestation des griefs en droit français de la concurrence: chronique d'un retour en Force », Revue juridique de l'économie publique, $\mathrm{n}^{\circ} 651$, mars 2008, étude 3; Casey William Hyland, et al., v. Homeservices of America Inc., Case n 3:05-cv-612 R, US district Court for the Western District of Kentucky, Louisville Division, 2007 U.S. Dist. Lexis 47503; 2007-2 Trade Cas (CCH) p. 75; D. Dukes, Individually and as Special Administratrix of the Estate of Merlon Dukes v. Pneumo Abex Corporation, Illinois Central Railroad, Metropolitan Life Insurance Company, and Owwens Illinois, and Honeywelle International Inc, $\mathrm{n}^{\circ}$ 4-06-0235, 386 III. App. 3d 425; 900 N.E. 2d 1128; 2008 III. App. Lexis 1252; 326 III. Dec. 881, disponible sur :

http://www.state.il.us/court/opinions/appellatecourt/2008/4thdistrict/october/4060235.pdf.

${ }_{74}$ B. Lasserre, Intervention au Colloque précité, Gazette du Palais, n 287-288, 14/15 octobre 2005.
} 
La non-contestation des griefs $^{75}$ ou procédure non contendere ne sont pas de véritables transactions mettant fin aux poursuites (plea bargaining). Il ne s'agit pas non plus d'une véritable alternative à des sanctions. En effet, cette procédure s'ouvre dans l'hypothèse où l'autorité de concurrence n'a pas encore qualifié les comportements ${ }^{76}$. Elle fait état néanmoins de «préoccupations concurrentielles» ${ }^{77}$ auxquelles les entreprises peuvent mettre fin en ne contestant pas la réalité des «griefs», qui ne sont cependant même pas qualifiés juridiquement d'infraction ${ }^{78}$. Même si formellement les entreprises en France n'ont plus l'obligation de prendre des engagements, substantiellement, l'objet de la procédure, qui est d'amener les entreprises à se conformer à une concurrence praticable telle que voulue par l'Autorité de la concurrence, conduit ces dernières à systématiquement en proposer.

Le véritable pouvoir de transaction qui met fin aux poursuites résulte de la procédure d'acceptation des engagements, née en droit communautaire et adoptée en France en 2004 (codifiée à l'article. L 462-2-I du Code de commerce ${ }^{79}$ ). Le Conseil a précisé que les «engagements doivent être adéquats pour remédier aux préoccupations de concurrence, avoir un contenu précis, être crédibles, réalisables rapidement et montrer la volonté de l'entreprise de ne plus entraver le libre jeu de la concurrence».

La procédure de clémence stricto sensu ne joue que pour les ententes ${ }^{81}$. Elle prévoit une exonération totale ou partielle à une entreprise qui a contribué à établir la réalité d'une entente et à identifier les auteurs en apportant les informations dont les autorités de concurrence ne disposaient

\footnotetext{
${ }^{75}$ Article L 462-2-III du Code de commerce.

${ }^{76}$ Notons qu'à la différence de la clémence stricto sensu qui ne joue qu'en matière d'entente, cette procédure est utilisable pour toutes les pratiques anticoncurrentielles (ententes et abus de domination, abus de dépendance économique : Aut. conc., déc. $\mathrm{n}^{\circ}$ 10-D-08, 3 mars 2010 relative à des pratiques mises en oeuvre par Carrefour dans le secteur du commerce d'alimentation générale de proximité, disponible sur :

http://www.autoritedelaconcurrence.fr/pdf/avis/10d08.pdf ; L. Boy, « Abus de dépendance économique: reculer pour mieux sauter? », Rev. Lamy Concurrence, avril/juin2010, p. 93), y compris aux Etats-Unis. Cartel de l'acier : CA Paris 19 janvier 2010, Pôle 5, ch. 5-7, n RG: 2009/00334, Revue Lamy de la concurrence, $\mathrm{n}^{\circ}$ 23, avril/juin 2010, p. 21, note L. Boy; E. Combe, C.Monnier, «Cartels in Europe : Main Features », Concurrences, n ${ }^{\circ}$ 2-2010, pp. 23-31; C. Sarrazin, «Compétence du Conseil de la concurrence : La Cour d'appel de Paris confirme la compétence du Conseil de la concurrence dans l'affaire du cartel du négoce des produits sidérurgiques (AMD Sud-Ouest) », Concurrences, $n^{\circ} 2$ 2010, pp. 71-72; M. Debroux, «Rôle du plafond des sanctions : La Cour d'appel de Paris confirme la compétence du Conseil de la concurrence et sa qualification des faits dans l'affaire du négoce des produits sidérurgiques, mais réduit fortement la sanction, au motif d'une atteinte « moyennement grave » à l'économie et sur le fondement d'une lecture surprenante du rôle du plafond des sanctions (AMD Sud-Ouest) », Concurrences, $n^{\circ}$ 2-2010, pp. 68-70.

${ }^{77}$ Nolo contendere est l'expression latine pour "I do not wish to contend". When using "nolo contendere" firms don't recognize any violation of the law or any civil fault.

${ }^{78}$ L'article L. 464-2 du Code de commerce français dans sa version antérieure à l'entrée en vigueur de la loi $\mathrm{n}^{\circ} 2008$ 776 du 4 août 2008 de modernisation de l'économie disposait que le Conseil de la concurrence pouvait «accepter des engagements proposés par les entreprises ou organismes et de nature à mettre un terme aux pratiques anticoncurrentielles». La formulation pouvait amener à penser que le Conseil devait qualifier les pratiques litigieuses. Ce n'est donc plus le cas aujourd'hui. La procédure d'engagement présente des spécificités par rapport à la procédure habituelle. Elle ne comporte plus qu'une simple évaluation préliminaire des pratiques en vue d'identifier des préoccupations de concurrence, sans qu'il soit donc nécessaire de procéder à la qualification de comportements au sens des articles L. 420-1 ou L. 420-2 du Code de commerce ni, a fortiori, à la constatation d'une infraction à ces dispositions (Paris, 16 oct. 2007, BOCC, 15 sept. 2008 ; D. 2007, AJ. 2729, obs. Chevrier; CCC 2007, n 297, obs. Malaurie-Vignal ; Gaz. Pal. 2008, 2692, et les obs.; RJDA 2007, n 1282 ; CCE 2007, ${ }^{\circ} 152$, obs. Chagny; RLC janv.-mars 2008. 85, note Cheynel); E. Chevrier, «Préoccupations de concurrence en matière d'engagements », $D$. Actualité, 26 mars 2009.

${ }^{79}$ Article 10 de l'ordonnance ${ }^{\circ}$ 2004-1173 du 4 nov. 2004.

${ }^{80}$ Cons. conc., déc. $n^{\circ} 05-\mathrm{S}-01$ et $05-\mathrm{D}-12$.

${ }^{81}$ B. Lasserre, «La politique des engagements en matière de pratiques anticoncurrentielles : premiers pas et premier bilan en France », contribution à la 32e conférence International Antitrust Law and Policy, à l'Université de Fordham, disponible sur: http:// www. conseil-concurrence. fr/ doc/ engagements_lasserre_fordham.pdf; F. Mélin, Les programmes de clémence en droit de la concurrence, Droit français et droit communautaire, Joly éditions, 2010; Art. L 462-2-IV du Code de commerce; J.-S.Bergé, «Le droit européen et la clémence », intervention au colloque $L a$ clémence et le droit, Le Havre, nov. 2009, disponible sur : http://www.cejec.eu/wpcontent/uploads/2010/01/contribution-jsberge-colloque-clemence-et-le-droit2.pdf.
} 
pas antérieurement. Avec des variantes ${ }^{82}$, la clémence se conjugue généralement en clémence de premier rang et de second rang selon l'importance de l'apport fourni aux autorités ${ }^{83}$.

Quelles que soient les procédures et leurs qualificatifs, ces diverses techniques traduisent moins un simple phénomène d'acculturation juridique imposé par les Etats-Unis que la reconnaissance de nouvelles représentations communes fondées sur une nouvelle morale utilitariste ${ }^{84}$, répondant à des objectifs d'efficacité et d'efficience ${ }^{85}$.

Pour mesurer ces dernières, un examen rapide des engagements s'impose. Généralement, l'accord «global» constitue une sorte de contrat cadre organisant des engagements diversifiés : engagements structurels, engagements comportementaux et quasi structurels. En schématisant, on peut dire que, par l'acceptation des engagements, les autorités et les entreprises co-organisent la concurrence en remodelant les contrats ${ }^{86}$. Le premier constat que fait généralement la doctrine est le succès rapide ${ }^{87}$ de ces diverses procédures ; même si une bonne partie de celle-ci, constituée principalement de praticiens, les critique en partie au nom de l'insécurité juridique qu'elles auraient engendrée ${ }^{88}$. Pour répondre à ces critiques souvent excessives, les autorités de concurrence ont publié des guidelines ou des communiqués divers de procédures ${ }^{89}$.

Il est devenu banal de parler en termes généraux d'engagements, voire de remèdes, et de se référer à la classification structure/comportement. Apparus en matière de contrôle des concentrations, les engagements sont très souvent structurels, notamment via des cessions d'actifs non stratégiques et de titres $(\text { carve out })^{90}$, ou quasi structurels, comme la résiliation de contrats exclusifs de

${ }^{82}$ Il faut noter que le DOJ (USA) dispose d'un pouvoir exclusif de rompre les négociations à la suite du Tunney Act (15 U.S.C. § 16). Le DOJ a parfois utilisé ce pouvoir pour contraindre les entreprises, mais les signaux du DOJ avant l'entrée dans les négociations sont souvent assez précis. Voir Branfman, «Antitrust Consent Decrees: A Review and Evaluation of the First Seven Years Under the Antitrust Procedures and Penalties Act», 27 Antitrust Bulletin, 1982, p. 303.

${ }^{83}$ On parle de «valeur ajoutée significative».

${ }^{84}$ Y. Chaput, «Philosophie des programmes de clémence et de transaction», colloque Clémence et transaction précité (note 2), p. 16.

${ }^{85}$ G. Canivet, M-A Frison-Roche et M. Klein (dir.), Mesurer l'efficacité économique du droit, LGDJ, coll. Droit et économie, 2005 ; Federal Trade Commission, A Study of the Commission's Divestiture Process, août 1999, disponible sur : http://www.ftc.gov/os/1999/08/divestiture.pdf; A. Winckler, «L'efficacité curative des décisions en droit de la concurrence », in L'efficacité des décisions en matière d'ententes et de concentrations, ateliers DGCCRF08/09/2003, disponible sur :

http://www.minefi.gouv.fr/fonds_documentaire/dgccrf/02_actualite/ateliers_concu/decisions.htm.

${ }^{86}$ L. Idot: "Les premières décisions acceptant des engagements, qu'elles soient adoptées par la Commission et le Conseil de la concurrence, confirment l'intérêt de cette technique de "remodelage souple" des contrats qui soulèvent des problèmes de concurrence», «Le régime des contrats en cours de formalisation », op. cit., p. 697; N. JalabertDoury, L. Nouvel, N. Mouy, T. Hoehn, Dr Ma. Gaved, F. Amand, Th. Piquereau, « Le suivi des engagements souscrits dans le cadre du contrôle des concentrations en France », Concurrence, 2007, n 2 et 4, p. 29 et p. 36.

87 A. Nuzzi, G.-B Nuzzi, "Main Trends of the Italian Competition Authority's Activity: A Law and Economics Perspective", European Business Law Review, 2008, Vol. 19, Issue 5. Examinant plusieurs affaires traitées par l'Autorité italienne, dont l'affaire ENI, les auteurs écrivent: "this strategy seems, in addition, perfectly consistent with the evolution of the European Commission approach, characterized by an extensive application of the 'commitments' provision of EC Regulation».

${ }^{88}$ Le recours aux prétendues violations de l'article 6 de la CEDH semble devenue quasiment un argument «de style» de la part des avocats, au point que les discussions sur ce sujet sont désormais parfois l'essentiel du texte des décisions, au détriment du fond, ce qui devient «irritant». On pense notamment à l'affaire de l'entente dans la téléphonie mobile: Cour cass., 29 juin 2007, Lamy droit des affaires, oct. 2007, n²0, p. 41, note L. Boy.

${ }^{89}$ Par exemple, le Conseil de la concurrence a publié, le 3 avril 2008, un communiqué de procédure relatif aux engagements en matière de concurrence, destiné à synthétiser la pratique de décision en la matière :D. actualité, 9 avril 2008.

${ }^{90}$ D. L. Feinstein, M.B. Bernstein, “All Over the Map: Grocery Store Enforcement from Von's to Whole Foods”, Antitrust, Chicago, Fall 2007, Vol. 22, Issue 1, p. 52. Pour de nombreux exemples en droit américain: R. B. Keiner, Jr., L. B. Halloway and G. F. Murphy, 1 Crowell \& Moring LLP, Airline Alliances, Antitrust Immunity and Mergers in the United States, disponible sur : http://www.crowell.com/documents/airline-alliances-antitrust-immunity-and-mergers-inthe-us.pdf ; Comm. CE, 11 déc. 2006, La Commission autorise le projet d'acquisition de l'activité de Pfizer dans le domaine des produits de soins par Johnson \& Johnson, sous réserve de certaines conditions, IP/06/1726, 11.12.2006. 
distribution $^{91}$, de licence, d'enseigne ${ }^{92}$. Ces engagements seraient, en effet, préférés par les autorités dans la mesure où il serait plus facile pour ces dernières d'en assurer le suivi et donc leur effectivité. L'affirmation mérite cependant d'être nuancée. Du fait du recours de plus en plus fréquent aux engagements, les autorités de concurrence aussi bien que les entreprises ont acquis une maîtrise toujours plus sophistiquée de ces derniers, et recourent souvent à des montages subtiles associant engagements structurels, comportementaux et quasi structurels $(E . O N)^{93}$. C'est ainsi que l'entreprise peut s'engager à maintenir une comptabilité distincte entre produits concurrents ${ }^{94}$, ouvrir un accès à ses infrastructures essentielles ${ }^{95}$, renoncer à un effet anti-concurrentiel lié à un comportement tel que des remises de gamme (SEB/Moulinex) ou autre ${ }^{96}$. Quoiqu'il en soit de tous ces types d'engagements, les possibilités qu'ils offrent sont quasi infinies, et leur suivi soulève la question délicate du contrôle des contrats économiques dans lesquels ils s'insèrent, question liée, bien sûr, à leur nature originale. Il ne faut pas oublier, en effet, que les obligations des autorités de concurrence de réduire les sanctions encourues ou de ne pas poursuivre, voire de ne pas qualifier d'infractions les comportements des entreprises viennent en compliquer l'analyse.

\subsection{Une nature complexe ordonnée autour du facteur temps}

Depuis longtemps déjà la doctrine a relevé, en étudiant tel ou tel contrat économique, qu'il soulevait de délicats problèmes de qualification. Il suffit de rappeler les difficultés de qualification de certains

\footnotetext{
91 Affaire Boeing/MC Donnell Douglas. L'UE a fini par autoriser la fusion le 30 juillet 1997. Boeing a renoncé aux «contrats d'exclusivité» qu'il avait conclus auparavant avec trois compagnies américaines (Continental, Delta et American) aux termes desquels ces dernières s'engageaient à se fournir uniquement chez le constructeur de Seattle. Boeing s'était aussi engagé à ne plus négocier à l'avenir de tels contrats, A. Gosset-Grainville, « Le cadre juridique de la détermination et de la mise en oeuvre des remèdes en France et en Europe », L. Benzoni, «L'analyse économique dans la détermination des remèdes: démarches, apports, limites », in Les remèdes dans les opérations de concentration, Ateliers de la Concurrence - DGCCRF, 7 mars 2007, disponible sur:
}

http://www.teraconsultants.fr/assets/publications/PDF/2007-Mars-

Les_remedes dans les_operations de concentration-Benzoni-Ateliers de la_Concurrence.pdf; C. Esteva, St. Hautbourg et A. Grémillet, «Intervention introductive: enseignement de l'analyse ex post des remèdes par la Commission européenne », Atelier de la concurrence, 7 mars 2007, Concurrence et consommation, $\mathrm{n}^{\circ}$ 156, déc. 2007; Théophile et Parmentier, «L'étendue du contrôle juridictionnel dans le contentieux du contrôle des concentrations en droit français et communautaire », Concurrences, $\mathrm{n}^{\circ} 1-2006, \mathrm{n}^{\circ} 463, \mathrm{p} .39$ et $\mathrm{s}$.

92 Autorité Conc. n 09-D-016, 22 juin 2009 relative à la fusion entre les groupes Caisse d'Epargne et Banque populaire, disponible sur: http://www.autoritedelaconcurrence.fr/pdf/avis/09d16.pdf; plus emblématique, l'affaire Moulinex/Seb II, 16 août 2004. Remise en cause par le Conseil d'Etat quelques temps plus tôt, l'autorisation de la reprise partielle de l'inventeur du moulin à légumes par le leader mondial du petit électroménager avait été finalement autorisée, Bercy ayant donné son feu vert après que l'entreprise ait pris un certain nombre d'engagements; Comm. CE, 18 juillet 2007, SFR/Télé 2 France, aff. COMP/M/ 5404 (Autorisation en phase II, sous réserve d'engagements comportementaux).

93 Affaires E.ON et RWE, L. Idot, (De quelques illustrations des limites de la distinction entre le comportemental et le structurel) : Comm. CE, 26 novembre 2008, aff. 39/388 et 39/389, Marché de gros de l'électricité en Allemagne et marché de l'équilibrage en Allemagne, résumé publié, JOUE, 13 févr. 2009, n ${ }^{\circ}$ C36; Comm. CE, 11 mars 2009 , aff. 39/442, RWE, résumé publié, JOUE, 12 juin 2009, n ${ }^{\circ} \mathrm{C} 133$, Revue des contrats, 1er juillet $2009 \mathrm{n}^{\circ} 3$, p. 1070, Aut. conc., 24 nov. 2009, Secteur de la viande de volaille: engagement de cession d'actifs sur le marché de la production et de la commercialisation de viande de volaille fraîche et engagements de ne plus subordonner les remises au référencement sur le marché des produits élaborés. Voir aussi: A. Winckler, «L'efficacité curative des décisions en droit de la concurrence », op. cit.

${ }^{94}$ Comm. CE, 2 sept. 2003, GE/instrumentarium, JOUE, ${ }^{\circ}$ L.109, 16 avril 2004.

95 Aut. conc., $\mathrm{n}^{\circ}$ 10-DCC-11, 26 janvier 2010, relative à la prise de contrôle exclusif par le groupe TF1 des sociétés NT1 et Monte-Carlo Participations (groupe AB), disponible sur :

http://www.autoritedelaconcurrence.fr/pdf/avis/10DCC11decisionversionpublication.pdf. TF1 s'est engagée à prendre toutes les mesures de nature à permettre une meilleure circulation portant sur les œuvres patrimoniales d'expression originales françaises et cinématographiques de manière à faciliter l'accès à ces droits sur les seconds marchés.

${ }^{96}$ Aut. conc., 26 janvier 2010, Rachat par TF1 des chaînes TMC et NT1 (op. cit.). Cette décision montre la richesse des engagements possibles. Voir entre autres: ne pas répondre à des appels d'offres, ne pas pratiquer de couplage en contrepartie d'espaces publicitaires. 
contrats de dépendance, révélées à propos des difficultés de la détermination du prix ${ }^{97}$. S'agissant des contrats d'union et plus spécialement des ententes, la doctrine n'est même pas d'accord pour en faire une variété de contrats innommés ${ }^{98}$. Ces dernières sont révélatrices de la spécificité des contrats économiques. Mais les problèmes les plus importants pour des raisons évidentes - la présence d'une partie étatique - ont été rencontrés avec les contrats de souveraineté. On a pu contester d'abord leur qualification même de contrats. Lorsqu'on l'admet cette nature contractuelle, on discute en France du point de savoir s'il s'agit de contrats de droit commun ou de contrats administratifs, voire de situations contractuelles dans lesquelles prennent place des mécanismes unilatéraux $^{99}$ ou l'inverse ${ }^{100}$. Ces discussions très sensibles en France - dualité des juridictions oblige ! - ne doivent pas masquer l'essentiel qui est que l'on est en présence de mécanismes négociés dont il importe de relever les spécificités.

Observons d'abord avec G. J. Martin que, à la différence des contrats classiques pour lesquels le contrôle essentiel porte sur le consentement, l'équilibre du contrat ou la conformité à la morale par le biais de la cause immorale et illicite, en ce qui concerne les contrats économiques, le contrôle du consentement est quasi inexistant. Quant au contrôle sur le contenu, c'est très rarement un contrôle de l'équilibre interne du contrat, pour cette raison simple que le déséquilibre d'un contrat économique, sauf abus manifeste ${ }^{101}$, fait partie de sa nature et qu'un contrôle est difficilement concevable.

Finalement, ce qui frappe dans les contrats économiques c'est ce que l'on a pu les qualifier de "fragilisation de l'effet obligatoire» ${ }^{102}$. Il nous semble plus exact de relever l'importance du facteur temps en ce qui les concerne, du fait qu'ils se veulent des contrats cadres, des contrats organisateurs, et cet effet se manifeste jusques y compris dans le contentieux.

Contrats dont l'objet même est l'organisation des rapports économiques par la concentration et/ou la planification privée ou publique, la "matière" sur laquelle porte ces contrats est fluctuante par nature car soumise aux évolutions des conjonctures économiques et des politiques économiques. Ainsi l'a-ton vu pour les politiques de concurrence où ce qui compte aujourd'hui serait la recherche de l'efficacité. Ces contrats doivent s'adapter aux changements, raison pour laquelle même ces contrats de droit privé en France contiennent toujours des clauses d'adaptabilité, des clauses de hardship ${ }^{103}$. L'importance économique de ces accords conduit à «naviguer» entre variabilité temporelle (renégociabilité) et recherche d'une stabilité relative (neutralisation du pouvoir d'Etat par les nombreuses clauses de stabilisation). Au fond, ils obéissent à une certaine variabilité temporelle (adaptabilité, suivi, re-négociabilité, stabilisation et non stabilité).

Ce qui est remarquable, c'est que le facteur temps est essentiel aussi dans les décisions administratives ou quasi juridictionnelles des autorités de concurrence auxquelles donnent lieu les «engagements» en droit de la concurrence. Il y a des années déjà, A. Pirovano avait mis en lumière

\footnotetext{
${ }^{97}$ Pendant longtemps, la jurisprudence les a assimilé à des contrats de vente et les soumettait aux contraintes de l'article 1591 du Code civil. Ce n'est qu'à partir de 1978, qu'elle accepte d'y voir des contrats «spéciaux», tout en décidant de les soumettre à l'article 1129 du Code civil et de contrôler éventuellement l'abus dans la fixation du prix.

${ }^{98}$ Notamment, J. B. Blaise, Ententes et concentration économique, Sirey, 1983, spéc. p. 28.

${ }^{99}$ C. L. Vier, Le procédé contractuel dans l'administration économique, thèse Paris, 1972. Statut de fonctionnaire, par exemple. V.

D. Linotte et R. Romi, Services publics et droit public économique, Litec, 2001, p. 355 et s.

${ }^{101}$ On pense à l'abus de dépendance économique et aux déceptions qu'il a suscitées: A. Pirovano et M. Salah, « L'abus de dépendance économique, une notion subversive? », Les Petites Affiches, 21/24 sept. 1990, p. 42 ; L. Boy, « Abus de dépendance économique: reculer pour mieux sauter? », sous Aut. conc., déc. n $10-\mathrm{D}-08,3$ mars 2010, relative à des pratiques mises en oeuvre par Carrrefour dans le secteur du commerce d'alimentation générale de proximité, Rev. Lamy concurrence, $\mathrm{n}^{\circ} 23$, avril/juin 2010, p. 93.

102 G. J. Martin: "Cette fragilisation provient de causes diverses, variables selon les contrats. Elle est prouvée (négativement) par l'apparition de solutions ou de techniques "nouvelles" destinées précisément à lutter contre cette tendance. C'est dire que cet effet obligatoire fragilisé dans le cadre classique est en quelque sorte reconstitué par des voies originales», Cours précité. Outre l'abus de dépendance économique dont les résultats sont bien décevants, il faut faire état $\mathrm{du}$ droit des pratiques individuelles ou restrictives de concurrence du droit français (titre IV du Code de commerce) ou du droit allemand: refus de vente, pratiques discriminatoires...

${ }^{103}$ Les «nécessités économiques» font sauter les verrous traditionnels, en particulier le refus de la théorie de l'imprévision. A. Pirovano (dir.), Changement social et droit négocié, op. cit., p. 130.
} 
dans l'étude du caractère négocié du plan judiciaire de redressement de l'entreprise, les «deux caractéristiques de ce contrat complexe qu'est le plan négocié: stabilité et plasticité»» ${ }^{104}$.

Pour assurer ces qualités, ce sont moins les décisions elles-mêmes qui comptent que leur suivi ${ }^{105}$. En effet, la pratique a démontré que, même les décisions qui semblaient soulever le moins de difficultés: celles approuvant des engagements structurels, étaient en réalité beaucoup plus délicates à mettre en oeuvre qu'il ne le semble. Parmi les remèdes (remedies, undertakings ou commitments) proposés par les entreprises ou fortement suggérés à ces dernières, les cessions d'actifs qui sont donc des décisions a priori radicales ont fait l'objet d'études précises par les économistes. Il est banal en effet de lire que les autorités de concurrence préfèrent de loin les engagements structuraux ou quasi structuraux aux engagements comportementaux, notamment parce qu'ils seraient beaucoup plus faciles à surveiller ${ }^{106}$. Or, même dans ce cas, la réalité est beaucoup plus complexe ${ }^{107}$. En effet, de délicates questions se posent. L'une d'elles concerne le choix du repreneur. Celui-ci n'est pas encadré par les textes ${ }^{108}$, ce qui laisse une grande liberté aux parties concernées. Si l'entreprise qui mène l'opération est libre de proposer le nom de l'acquéreur des actifs cédés (il n'est pas exigé une détermination $a b$ initio de l'acquéreur (up front buyer), les autorité de la concurrence veillent à l'indépendance de ce dernier au regard de l'entreprise, de même qu'elles vérifient la viabilité de 1 'entreprise qui reprend les actifs cédés ${ }^{109}$. La question du périmètre des actifs cédés est fondamentale, et la responsabilité des autorités de concurrence est lourde car elles doivent s'assurer de l'indépendance de l'acquéreur ${ }^{110}$ mais aussi du fait que celui-ci a la capacité de développer l'entreprises désinvestie de façon concurrentielle, d'autant que pendant une période transitoire, le risque est grand de voir les actifs se dégrader ${ }^{11}$. Enfin, les autorités de concurrence essaient d'adopter une optique pragmatique qui conduit à nuancer l'opposition engagements structurels et comportementaux. Ainsi dans les cas d'abus potentiels de pouvoir de marché, elles distinguent généralement par des analyses assez fines les hypothèses de concentration d'entreprises capitalistiques, comme le sont celles de l'énergie où les remèdes sont plutôt structurels, des concentrations d'entreprises du secteur tertiaire où le capital est surtout immatériel, où les remèdes sont alors plutôt de type comportemental ou mixte (ouverture d'accès aux infrastructures, ou aux licences, suppression de relations d'exclusivité). Il est donc devenu fréquent de nommer un mandataire (trustee) pour s'assurer du suivi et du respect des engagements tant structurels que comportementaux $^{112}$, et il semble même que ce soit devenu une véritable profession ${ }^{113}$.

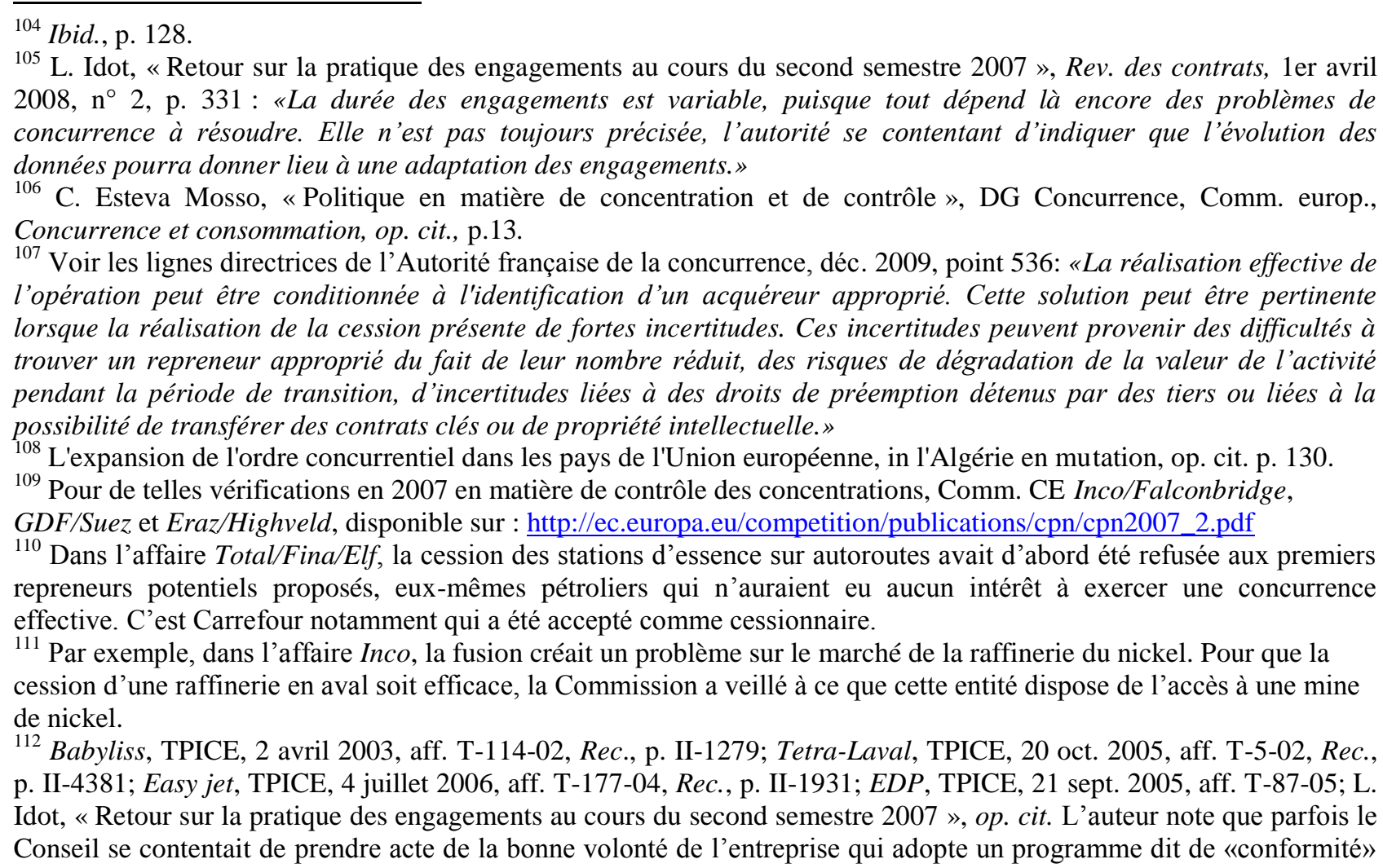


Dans cette matière quasi juridictionnelle ${ }^{114}$, les autorités construisent souvent le droit sur la base de standards (préoccupations de concurrence, concurrence praticable, engagements de nature à faire cesser...) et le font appliquer par le consensus ${ }^{115}$ qu'elles parviennent à réaliser autour de leur décision notamment après un test de marché (publication des propositions d'engagements sur leur site Internet dans le but de recueillir les observations des tiers intéressés) ${ }^{116}$. Ainsi que nous l'avons relevé, elles restent, après que des mesures aient été suggérées ou ordonnées, en charge des intérêts en cause et peuvent adapter leur décision ${ }^{117}$. Soucieuses d'une action pédagogique plus que d'une action répressive, elles préfèrent le suivi d'une conciliation au couperet de la décision ou du jugement.

Le contentieux, strictement entendu, des décisions rendues en matière d'engagements en droit de la concurrence est encore mal étudié et mériterait sans doute une recherche synthétique. On relèvera néanmoins une tendance à une exigence accrue en matière de motivation ainsi qu'un contrôle de proportionnalité $^{118}$ afin de donner un véritable contenu aux standards et ne pas tomber dans l'arbitraire. Il y va, en effet, de la crédibilité des engagements en droit de la concurrence ${ }^{119}$.

Mi-contrats, mi-décisions ou actes quasi juridictionnels, les décisions en matière d'engagements au sens large ne semblent toujours pas avoir reçu de qualification qui fasse l'unanimité. Au fonds peu importe leur qualification juridique surtout si l'on doit se référer à des catégories fondées aux XIX et XXémes siècles. Ces décisions sont bel et bien basées sur des mécanismes de concertation et de consentement plus ou moins libre, et illustrent la catégorie des contrats économiques de souveraineté telle que nous avons tenté d'en présenter l'analyse.

Il nous semble inopportun aujourd'hui de vouloir ramener un mécanisme très complexe à un schéma ancien qui propose des catégories nécessairement alternatives, formelles et unitaires (contrat ou décision ou jugement). Nous sommes en face de décisions administratives ou quasi juridictionnelles, négociées en tous cas dans leur élaboration comme dans leur exécution, qui s'étalent dans le temps même lorsque les engagements sont structurels. Les garanties de leur exécution résident en grande partie sur cet aspect conventionnel, lequel opère vraisemblablement un

(compliance program) ou met en place un mécanisme de surveillance interne (formation de personnel, mécanisme interne de whistleblowing (lanceur d'alerte); Ch. Lemaire, (A propos des programmes de conformité: quels enseignements à retirer de la pratique des autorités de concurrence à travers le monde), Concurrences, 2008, nº 1, p. 64; M. Debroux et R. Sainte Fare Garnot, «Programmes de conformité et risque concurrence: témoignages d'un juriste d'entreprise et d'un avocat », Concurrences, 2009, n², p. 230.

${ }^{113}$ Cela évoque le commissaire à l'exécution du plan dans les procédures collectives, J.M. Le Corre, Droit et pratiques des procédures collectives, Dalloz Action, 4éme éd. 2008/2009.

${ }^{114}$ D. Danet, «Le Conseil de la concurrence: juridiction incomplète ou juridiction innomée? », RIDE, 1991, n 1, p. 3; «Les magistratures économiques », RIDE, numéros spéciaux 2 et 3, 1997.

${ }^{115}$ L. Boy, «Les formes de traitement juridictionnel du contentieux économique », in A. Pirovano (dir.), Changement social et droit négocié, op. cit., p. 33.

116 J. Gstalter, «La Commission européenne et le Conseil de la concurrence consultent les tiers intéressés sur des propositions d'engagements », Concurrences, 2008, n³, p. 134.

${ }_{117} \mathrm{Ph}$. Gérard, F. Ost et M. Van de Kerchove, Fonction de juger et pouvoir judiciaire, Bruxelles, Publications des Facultés Universitaires de Saint-Louis, 1983, p. 47.

${ }^{118}$ L. Idot, «Retour sur la pratique des engagements au cours du second semestre 2007 », op. cit., p. 335 : «Le juge peut opérer un contrôle de l'adéquation des engagements à résoudre les problèmes de concurrence identifiés et plus encore sur la proportionnalité des engagements »; G. A Sofianatos, Injonctions et engagements en droit de la concurrence Etude de droit communautaire français grec, L.G.D.J., 2009; P. Nihoul, Ch. Verdure, Droit de la concurrence: aspects belge et européen, Bruxelles, Larcier, 2005.

119 A cet égard, une étude particulière devrait être menée sur les dangers de l'arbitrage dans le suivi des engagements. Bien que la Commission européenne favorise l'utilisation des clauses d'arbitrage, surtout en ce qui concerne le suivi des engagements comportementaux (Ch. Liebscher, «L'arbitrage dans les procédures de contrôle des concentrations: des perspectives », Gaz. Pal., 29 mai 2003, n 149, p. 24), il semble que les dangers soient grands d'un non-respect de l'ordre public concurrentiel (L. Idot, «Une innovation surprenante: l'introduction de l'arbitrage dans le contrôle communautaire des concentrations », Rev. Arb. 2000, p. 591; W. Abdelgaward, Arbitrage et droit de la concurrence, LGDJ, 2001, § 1159). 
retour aux racines du contrat: la réciprocité et la confiance, la reliance ou le concept d'attente légitime $^{120}$.

Summary: Economic contracts of sovereignty as competition regulation tools (the practice of the competition authorities with France as a case study)

In a globalized market economy, concentration and economic organization are the result of two combined elements: private economic power's strategies and public policy instruments of competition law. Over the past decade, the US and the EU competition law have undergone a range of substantive reforms. A wave of reforms has just been passed. These reforms, which will significantly remould the institutional architecture of the competition systems around the world, cover areas such as the promotion of private enforcement, settlements and leniency procedures for hardcore infringements, commitment decisions, market inquiries and informal regulations. In order to promote competitive markets, leniency programs and commitments are the new regulatory instruments used by competition authorities. Instead of sanctions and fines, competition authorities and judges prefer to use various procedural mechanisms based on commitments in order to fight hardcore cartel, abuses and to promote competitive markets. These arrangements constitute what can be termed as "economic sovereignty contracts". These new types of contracts haven't been thoughtfully analyzed yet in the scholarship. This paper analyzes the real consideration in these contracts, which is, as defined in Chinese law, " the purpose of realizing certain economic goals». To encourage a firm to confess or to stop an anticompetitive practise, enforcement agencies may promise a smaller fine, shorter sentences, less restrictive orders, or complete amnesty. In contract law, the mutual consideration is not only the different promises between the parties. It's the operation as a whole, which is the organization or planning of the economic power as a result of a merger or a reinforcement of private firms' power under the control of agencies, in accordance with economic public policy rules. Public economic rules may change in light of political concerns. Formally, States are sovereign. Substantially, they may choose to surrender part of their sovereignty; in the case of arbitration for instance. A part from these new economic contracts, leniency programs and commitments have a relative efficiency. With regard to these contracts, the question arises as to whether they are legally and fully binding. What can first be noticed is how important time and a proper enforcement mechanism are important in these types of contracts. Often, indeed, remedies proposed by firms to agencies need to be monitored; by a trustee for instance. Furthermore the enforcement mechanism used in these kinds of contract (trust or reciprocity) has an impact of the likelihood of their success.

Mots clés: droit de la concurrence, régulation, programmes de clémence, engagements, contrat économique, contrat de souveraineté, temps et contrat, confiance

Keywords: competition law, regulation, leniency programs, commitments, economic contract, sovereignty contract, time and contract, trust, reliance

Subject Descriptors (Econlit Classification System): K 12, K21, L 13, L 38, L 41, P 16

\footnotetext{
${ }^{120}$ J. Carbonnier, Flexible droit, Textes pour une sociologie du droit sans rigueur, LGDJ, 1969; B. Fauvarque-Cosson (dir.), La confiance légitime et l'estoppel, SLC, vol. 4, 2007; G. Gorla, Le contrat dans le droit continental et en particulier dans le droit français et italien, Turin, éd. de l'Institut universitaire d'études européennes, 1958 ; H. Muir Watt, «Reliance et définition du contrat », in Dialogues avec M. Jeantin, Dalloz, 1999, p. 57.
} 